\title{
Fatores que interferem na realização do aleitamento materno exclusivo
}

RESUMO | Objetivo: compreender a importância do aleitamento materno exclusivo (AME) e identificar os fatores que dificultam esse processo. Método: trata-se de uma revisão sistemática. As bases de dados consultadas foram a Literatura Latino-Americana e do Caribe em Ciências de Saúde, a Base de Dados Bibliográficos Especializada na Área de Enfermagem, a Medical Literature Analysis and Retrieval System Online e a Scientific Electronic Library Online. Resultados: a pesquisa resultou em 28 artigos, dos quais 11 atendiam a este estudo. A maioria dos fatores encontrados nos resultados (5) apresentou como fator de risco o uso de mamadeiras, a alimentação complementar e as chupetas; seguidos do grau de escolaridade das mães e de fatores socioeconômicos (4); do estado emocional das mães, do tipo de parto, de mães que trabalham fora e da falta de preparo dos profissionais (3). Outros fatores apareceram em menor ocorrência. Conclusão: o êxito do AME se faz com uma abordagem holística e efetiva dos profissionais de Enfermagem em todas as etapas da gestação: a concepção, o pré-natal e o puerpério.

Palavras-chaves: Aleitamento materno; Cuidado da criança; Lactação; Leite humano; Alimentação artificial.

\begin{abstract}
Objective: To comprehend the importance of exclusive breastfeeding (EBF) and identify factors that hinder this process. Methods: This is a systematic review. The databases used were the Latin American \& Caribbean Health Sciences Literature, the Bibliographic Database Specialized in Nursing, the Medical Literature Analysis and Retrieval System Online, and the Scientific Electronic Library Online. Results: The search produced 28 papers and 11 of them matched the objectives of this study. Most factors found in the results (5) showed risk, such as the use of feeding-bottles, complementary feeding and pacifiers. They were followed by the mothers' education levels and socioeconomic factors (4). After those were the mothers' emotional states, the type of delivery, the mothers working outside the home and the lack of preparation of the professionals (3). Other factors were present in smaller number of occurrences. Conclusion: The success of EBF is achieved through a holistic and effective approach of the nursing professionals in every pregnancy stage: conception, prenatal and puerperium.
\end{abstract}

Keywords: Breastfeeding; Child care; Lactation; Human milk; Artificial feeding.

RESUMEN | Objetivo: comprender la importancia de la lactancia materna exclusiva (LME) e identificar los factores que dificultan este proceso. Método: consistió enconsultas y en una revisión sistemáticade bases de datos como: Literatura Latinoamericana y Caribeña en Ciencias de la Salud, Base Bibliográficade Datos Especializados en el Área de Enfermería, Medical Literature Analysis and Retrieval System Online y Scientific Electronic Library Online. Resultados: la investigación descubrió 28 artículos, entro loscuales 11 se conformaban con los objetivosde este estudio. La mayoría (5) presentó, en cuanto factor de riesgo, el uso de biberones, complementos y chupetes; A continuación, apareció el grado de escolaridad de las madres y factores socioeconómicos (4); Por último, el estado emocional de las madres, la clase de parto, madres que trabajan fuera del hogar y la falta de preparación de los profesionales(3). Hay otros factores apuntados queson menos frecuentes. Conclusión: el éxito de la LME se logra bajo el abordaje holístico y efectivo de los profesionales de enfermería en todas las etapas del embarazo: concepción, prenatal y puerperio.

Palabras claves: Lactancia Materna, Cuidados con niños, Lactación, Leche Humana, Alimentación artificial.

\section{Andressa de Oliveira Rios Pereira}

Graduanda em Enfermagem, Universidade do Estado de Minas Gerais, Minas Gerais, Brasil.

ORCID: 0000-0001-9776-1938

\section{Raquel de Menezes Ferreira}

Graduada em Enfermagem, Universidade do Estado de Minas Gerais, Minas Gerais, Brasil.

ORCID: 0000-0003-0019-8494

\section{Fernanda Marcelino de Rezende e Silva}

Mestre em Enfermagem pela UFMG, Docente e Coordenadora do Curso de Graduação em Enfermagem da Universidade do Estado de Minas Gerais. Docente do Curso de Graduação em Enfermagem da Universidade de Itaúna (UIT).

ORCID: 0000-0003-2236-7009

\section{Karla Amaral Nogueira Quadros}

Enfermeira. Mestre em Educação, Cultura e Organização Social pela Universidade do Estado de Minas Gerais. Minas Gerais, Brasil. Docente do Curso de Graduação em Enfermagem da Universidade do Estado de Minas Gerais (UEMG), Unidade Divinópolis.

ORCID: 0000-0002-3750-4873

\section{Regina Consolação dos Santos}

Enfermeira. Mestre em Ciência pela Universidade Federal de São João Del Rei, Campus Centro Oeste Dona Lindu UFSJ - (CCO). Docente do Curso de Graduação em Enfermagem da Universidade do Estado de Minas Gerais (UEMG), Unidade Divinópolis.

ORCID: 0000-0002-7393-3210

Recebido em: 28/08/2020

Aprovado em: 09/11/2020

\section{Silmara Nunes Andrade}

Enfermeira. Doutora em Ciências da Saúde pela Universidade Federal de São João Del Rei, Campus Centro Oeste Dona Lindu UFS - (CCO). Docente do Curso de Graduação em Enfermagem da Universidade do Estado de Minas Gerais (UEMG), Unidade Divinópolis. ORCID: 0000-0002-1975-0827

\section{INTRODUÇÃO}

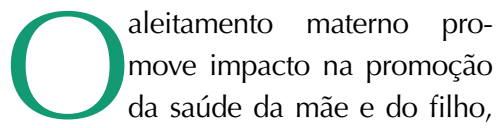
possibilitando, também, uma estratégia de vínculo entre ambos e sendo um fator de proteção e nutrição para a criança. Além disso, compõe a mais considerável e eco- 
nômica intervenção para a diminuição da morbimortalidade infantil. ${ }^{(1-2)}$

A Organização Pan-Americana da Saúde (OPAS) e a Organização Mundial da Saúde (OMS) destacaram que a amamentação é capaz de salvar vidas, no total, foram cerca de 820 mil crianças com idade inferior de cinco anos. Dados de 2017 mostraram que 78 milhões de recém-nascidos no mundo esperaram por mais de uma hora para serem amamentados pelas suas mães. A América Latina e o Caribe são as regiões com maior média de aleitamento materno. Na região das Américas, apenas $38 \%$ dos recém-nascidos são alimentados exclusivamente com o leite materno até os seis primeiros meses e $32 \%$ continuam a amamentação até os dois anos de idade. Estes dados são alarmantes e necessitam de uma atenção especial. ${ }^{(3)}$

Recomenda-se iniciar a amamentação nos primeiros 60 minutos de vida do bebê, pois há componentes imunológicos e probióticos do leite materno que protegem o bebê de infecções respiratórias e intestinais, evitam diarréias, diminuem o risco de alergias e previnem a perda de peso na primeira semana de vida, além de favorecer a criação de um laço afetivo entre mãe e filho. ${ }^{(4)}$

O Ministério da Saúde (MS) aconselha a amamentação de forma exclusivaaté os seis meses de idade da criança e de forma complementar até os dois anos, não sendo sugerida a ingestão de água, chás ou outros tipos de leites como forma complementar nos primeiros seis meses de vida, pois o uso dessas substâncias está diretamente relacionado ao desmame precoce, é entendida como a interrupção do aleitamento materno exclusivo ao peito, e a introdução de suplementos e complementares antes de o lactente completar seis meses de vida. ${ }^{(4-6)}$

$\mathrm{O}$ uso de formas e leites artificiais e chás podem trazer danos à saúde da criança, de modo que aumentem os riscos de infecções gastrointestinais, com maiores episódios de diarréia, que pode ser causada por água ou alimentos contaminados, propiciando novas enfermidades. ${ }^{(5)}$

O hábito da amamentação no Brasil começou na década de 1970, época em que ocorreu a sua grande queda, afetando alarmantemente a saúde das mães e das crianças. Houve, naquele período, muitas propagandas antiéticas relacionadas aos substitutos do leite materno, elevando o número de desmame precoce e aleitamento artificial. Aspectos culturais e sociais, interesses econômicos e a forma de enxergarem o ato de amamentar de forma não natural ocasionaram os altos índices de desnutrição e também de infecções recorrentes nos lactentes. ${ }^{(7)}$

Nesta perspectiva, a Política Nacional de Aleitamento Materno (PNAM), aprovada em 1999, é organizada de acordo com as seguintes estratégias: Incentivo ao Aleitamento Materno na Atenção Básica Rede Amamenta Brasil; Iniciativa Hospital Amigo da Criança (IHAC), Método Canguru na atenção hospitalar; Rede Brasileira de Bancos de Leite Humano; proteção legal através da Norma Brasileira de Comercialização de Alimentos para Lactentes (NBCAL); ações de mobilização social por meio de campanhas e parcerias; monitoramento das ações e práticas de aleitamento materno; e, nos últimos anos, implantação da iniciativa Unidade Básica Amiga da Amamentação. ${ }^{(8-10)}$

Ações de proteção e promoção do aleitamento materno necessitam de empenhos coletivos e dependem de vários fatores para se obter o sucesso, sendo um enorme desafio da saúde pública. Uma vez que é necessária a realização de uma abordagem humanizada e integral, os profissionais devem estar capacitados para acolher as mães tanto no pré-natal quanto no parto e no puerpério, pois em todas as fases vão surgir dúvidas e inseguranças. Por isso, os profissionais devem ressaltar, durante as consultas e ações educativas que serão desenvolvidas nesse período, a importância do aleitamento materno, enfatizando os seus benefícios e utilizando a comunicação e o acolhimento como ferramentas que possam conscientizar e apoiar as mães. ${ }^{(11)}$

A equipe de enfermagem devem estimular a participação da família neste processo e a formação de grupos de apoio às gestantes, podendo saciar dúvidas como o tempo ideal de aleitamento materno, as conseqüências do desmame precoce, a produção de leite, a amamentação nos primeiros minutos de vida, o alojamento conjunto, os direitos dos pais, as técnicas da amamentação e o incentivo ao parto normal, bem como acompanhar o processo de desenvolvimento da criança, seja por consulta individual ou visita domiciliar. ${ }^{(4)}$

Atualmente, ainda existem bastantes mitos, tabus e preconceitos sobre a amamentação ${ }^{(25)}$. Vários fatores também afetam o aleitamento materno exclusivo (AME), como a interferência familiar com antigas tradições, a falta de conhecimento em relação à posição e à prega correta, a falta de instruções pelos profissionais de saúde, fatores biológicos (como a produção de leite e a estrutura da mamas, tais como o mamilo plano e invertido), fatores econômicos, influências negativas e insegurança das mães. ${ }^{(12)}$

Este estudo contribuirá para o conhecimento dos fatores que interferem no AME e, assim, aprofundar no papel do enfermeiro na assistência prestada às mães, principalmente no pré-natal, já que a assistência nesta fase carrega um conjunto de procedimentos capazes de identificar antecipadamente esses dificultadores, que comprometem diretamente a oferta da lactação. A pesquisa buscou também colaborar para o sucesso do aleitamento materno e diretamente com a redução das taxas de mortalidade infantil, saúde física e mental e qualidade de vida das mães e dos bebês. ${ }^{(12-13)}$

Diante deste contexto, e acreditando na relevância do tema para a saúde do recém-nascido e da mãe, este estudo poderá oferecer a compreensão da importância do AME, bem como identificar os fatores que dificultam o processo do aleitamento materno.

\section{MÉTODO}

Trata-se de uma revisão sistemática da literatura que seguiu as recomendações do instrumento dos Principais Itens 
para Relatar Revisões Sistemáticas e Metanálise(PRISMA). ${ }^{(14)}$ A revisão sistemática é usada para possibilitar uma análise objetiva dos resultados, com o intuito de relataras evidências de uma determinada pergunta e onde esta será selecionada e avaliada,evitando os vieses que possam surgir. ${ }^{(15)}$ Para a identificação dos fatores, a busca das publicações foi realizada a partir da seguinte pergunta: "Quais são os fatores que influenciam no êxito do aleitamento materno exclusivo?".

A busca na literatura foi realizada a partir dos seguintes critérios de inclusão: textos completos disponíveis, no período dos últimos cinco anos, nos idiomas por- tuguês, inglês e espanhol, disponibilizados na íntegra, como tipo de documento modelo de artigo, e tendo como país de filiação o Brasil. As teses, as dissertações e os artigos que não respondiam à pergunta norteadora foram excluídos.

As bases de dados consultadas forama Literatura Latino-Americana e do Caribe em Ciências de Saúde (LILACS), aBase de Dados Bibliográficos Especializada na Área de Enfermagem (BDENF), a Medical Literature Analysis and Retrieval System Online (MEDLINE) e a Scientific Electronic Library Online (SciELO).

Os descritores utilizados foram os seguintes: "Aleitamento materno; Cuidado da criança; Lactação; Leite humano; Alimentação artificial". Eles foram cadastrados nos Descritores em Ciências da Saúde (DeCS). A busca nas bases de dados foi realizada em julho de 2019. Como resultado, foram obtidos 28 artigos no total, nas quatro bases de dados. Posteriormente, foi realizada a leitura dos títulos e dos resumos de cada artigo, com a intenção de identificar quais atendiam ao objetivo da pesquisa. Desta forma, foram selecionados 11 artigos, tabulados no software Microsoft Excel e apresentados em quadro sinóptico, contendo título, ano e periódico dos artigos. Dos 28 artigos, sete foram excluídos por não atenderem à pergunta norteadora,quatro por se-

\section{Quadro 1 - Artigos utilizados para o estudo pesquisados nas bases de dados, 2014-2019.}

\begin{tabular}{|c|c|c|c|}
\hline $\begin{array}{l}\text { Arti- } \\
\text { go }\end{array}$ & Objetivo do estudo & População do estudo & Fatores associados \\
\hline $1^{(16)}$ & $\begin{array}{c}\text { Determinar se existe uma relação } \\
\text { entre AMI, fatores natais, controle } \\
\text { pré-natal e características socioeco- } \\
\text { nômicas. }\end{array}$ & $\begin{array}{l}\text { Estudo de caso e controle. Foram aplicados } \\
\text { questionários às mulheres com filhos entre } 6 \text { e } \\
24 \text { meses de idade. }\end{array}$ & Fator socioeconômico e menor escolaridade. \\
\hline $2^{(17)}$ & $\begin{array}{l}\text { Verificar a associação entre a } \\
\text { depressão pós-parto e a ocorrência } \\
\text { do AME. }\end{array}$ & $\begin{array}{c}\text { A amostra para o estudo, ocorrido em uma ação } \\
\text { de multivacinação, consistiu em } 2.259 \text { pares de } \\
\text { mãe-filho. }\end{array}$ & $\begin{array}{l}\text { Depressão pós parto; mulheres mais jovens } \\
\text { tendem a interromper o AME primeiro; pré-natal } \\
\text { tardio; e fator socioeconômico (baixa e alta con- } \\
\text { dição socioeconômica é um fator de interrupção } \\
\text { do aleitamento materno). }\end{array}$ \\
\hline $3^{(18)}$ & $\begin{array}{l}\text { Analisar a valoração axiológica da } \\
\text { mulher/nutriz quanto ao manejo } \\
\text { clínico da amamentação. }\end{array}$ & $\begin{array}{c}20 \text { nutrizes, em alojamentos conjuntos de dois } \\
\text { hospitais universitários. }\end{array}$ & Insegurança após alta da maternidade. \\
\hline $5^{(20)}$ & $\begin{array}{l}\text { Analisar se as informações veicu- } \\
\text { ladas em sites populares estão de } \\
\text { acordo com os passos recomendados } \\
\text { no guia alimentar para crianças } \\
\text { menores de dois anos do MS. }\end{array}$ & $\begin{array}{c}\text { Estudo descritivo/comparativo, executado entre } \\
\text { agosto e outubro de 2014, no qual foi feita uma } \\
\text { busca em sites populares de língua portuguesa } \\
\text { que divulgaram informações sobre a alimenta- } \\
\text { ção de crianças menores de dois anos. Foram } \\
\text { analisados } 50 \text { sites, entre os quais blogs, sites de } \\
\text { empresas alimentícias e sites especializados em } \\
\text { nutrição infantil. }\end{array}$ & $\begin{array}{l}\text { Informações incoerentes e contrárias às reco- } \\
\text { mendadas no guia alimentar. }\end{array}$ \\
\hline
\end{tabular}




\begin{tabular}{|c|c|c|c|}
\hline $7^{(22)}$ & $\begin{array}{l}\text { Identificar a prevalência da ama- } \\
\text { mentação na primeira hora de vida e } \\
\text { seus resultados para a manutenção } \\
\text { do aleitamento materno. }\end{array}$ & $\begin{array}{l}\text { Trata-se de estudo descritivo e prospectivo, com } \\
\text { abordagem quantitativa, realizado no } 2^{\circ} \text { e } 3^{\circ} \\
\text { trimestres de } 2015 \text {, em uma instituição com a } \\
\text { IHAC de Foz do Iguaçu (PR),município de Tríplice } \\
\text { Fronteira, junto com Porto Iguaçu (Argentina) } \\
\text { e Cidade do Leste (Paraguai).No período de es- } \\
\text { tudo, aconteceram } 456 \text { nascimentos.Destes, } 88 \\
\text { foram avaliados na primeira e segunda etapas e } \\
73 \text {, na terceira. }\end{array}$ & $\begin{array}{l}\text { Não realização do aleitamento materno na } \\
\text { primeira hora de vida; tipo de parto; e uso de } \\
\text { complemento alimentar antes dos seis meses. }\end{array}$ \\
\hline $8^{(23)}$ & $\begin{array}{l}\text { Avaliar o impacto de atualização de } \\
\text { profissionais da Atenção Primária à } \\
\text { Saúde em relação ao guia alimentar } \\
\text { "Dez passos para uma alimentação } \\
\text { saudável para crianças brasileiras } \\
\text { menores de dois anos" nas práticas } \\
\text { de aleitamento materno e na quali- } \\
\text { dade da alimentação complementar } \\
\text { de lactentes assistidos por Unidades } \\
\text { de Saúde de Porto Alegre (RS) }\end{array}$ & $\begin{array}{l}\text { Lactentes assistidos por Unidades de Saúde da } \\
\text { cidade de Porto Alegre (RS). }\end{array}$ & $\begin{array}{l}\text { Uso de complementos alimentares antes dos } \\
\text { seis meses e falta de preparo dos profissionais. }\end{array}$ \\
\hline $9^{(24)}$ & $\begin{array}{l}\text { Identificar os fatores associados } \\
\text { à descontinuação do AME em um } \\
\text { município do Nordeste do Brasil. }\end{array}$ & $\begin{array}{c}\text { Este estudo de coorte envolveu } 1.344 \text { pares } \\
\text { de mãe-filho selecionados em maternidades } \\
\text { de Feira de Santana (BA). Os indivíduos foram } \\
\text { acompanhados por seis meses através de visitas } \\
\text { domiciliares mensais. Foi registrada a interrup- } \\
\text { ção do AME. }\end{array}$ & $\begin{array}{l}\text { Uso de complementos alimentares antes dos } \\
\text { seis meses; limitação à amamentação noturna; } \\
\text { lesões nos seios; falta de apoio e incentivo por } \\
\text { parte do pai ou parceiro; qualidade do pré-natal } \\
\text { insatisfatória; falta de preparo dos profissionais; } \\
\text { grau de escolaridade; mães que trabalham fora; } \\
\text { e estado emocional das mães. }\end{array}$ \\
\hline $10^{(25)}$ & $\begin{array}{l}\text { Desenvolver um escore de IAC e } \\
\text { estudar sua associação com variáveis } \\
\text { socioeconômicas, clínico-epidemioló- } \\
\text { gicas e nutricionais. Esta análise visa } \\
\text { a propor ações e estratégias para a } \\
\text { abordagem nutricional adequada da } \\
\text { criança durante o primeiro ano de } \\
\text { vida no âmbito da Atenção Primária. }\end{array}$ & $\begin{array}{l}\text { Trata-se de um estudo transversal de aborda- } \\
\text { gem qualiquantitativa que se deu a partir do } \\
\text { contato com a equipe da Unidade de Saúde } \\
\text { da Família Perseu Leite de Barros (USF-PLB), } \\
\text { localizada no distrito Noroeste do município de } \\
\text { Campinas (SP), e da formalização da parce- } \\
\text { ria com a Secretaria Municipal de Saúde. As } \\
\text { informações provenientes de um questionário } \\
\text { realizado com famílias com crianças menores de } \\
\text { seis anos foram o objeto de análise. }\end{array}$ & $\begin{array}{l}\text { Fator socioeconômico; grau de escolaridade; } \\
\text { mães que trabalham fora; tipo de parto; uso de } \\
\text { complementos alimentares antes dos seis meses; } \\
\text { culturas e crenças já vivenciadas pelas mães na } \\
\text { amamentação; e ausência de companheiros. }\end{array}$ \\
\hline $11^{(26)}$ & $\begin{array}{l}\text { Avaliar os determinantes ao abando- } \\
\text { no do AME. }\end{array}$ & $\begin{array}{l}\text { Estudo longitudinal baseado em coorte de } \\
\text { nascimentos realizada em Viçosa (MG). Acom- } \\
\text { panharam-se } 168 \text { parturientes provenientes da } \\
\text { rede pública de saúde em } 2011 / 2012 \text {. Foram } \\
\text { realizadas três entrevistas com as parturientes: } \\
\text { aos } 30,60 \text { e } 120 \text { dias após o parto. }\end{array}$ & $\begin{array}{l}\text { Uso de complementos alimentares antes dos } \\
\text { seis meses; gestação não planejada; experiências } \\
\text { estressantes no parto; depressão pós-parto; } \\
\text { grau de escolaridade; mães que trabalham fora; } \\
\text { falta de apoio e incentivo por parte do pai ou } \\
\text { parceiro; insegurança de amamentar após a alta } \\
\text { da maternidade;e falta da rede de apoio. }\end{array}$ \\
\hline & 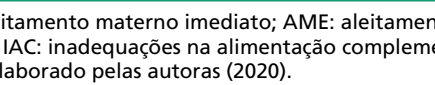 & & de vida; IHAC: Iniciativa Hospital Am \\
\hline
\end{tabular}

rem duplicados, três por serem relatos e três por não terem o texto completo disponível (Quadro 1).

A Figura 1 esquematiza o método percorrido de acordo com os critérios metodológicos.

\section{RESULTADOS}

Inicialmente, foram encontrados 28 artigos relacionados aos descritores controlados utilizados nesta revisão sistemática, porém, após os processos de seleção e elegibilidade, apenas 11 foram incluídos para a síntese final dos dados. Todos os estudos referentes aos textos foram conduzidos nos Brasil nos anos de 2014 a 2019.

Os fatores de risco descritos nos resultados desta revisão foram caracterizados como: falta de preparo dos profissionais; estado emocional da mãe; idade materna 
Figura 1 - Fluxo de informação da revisão sistemática, 2019.

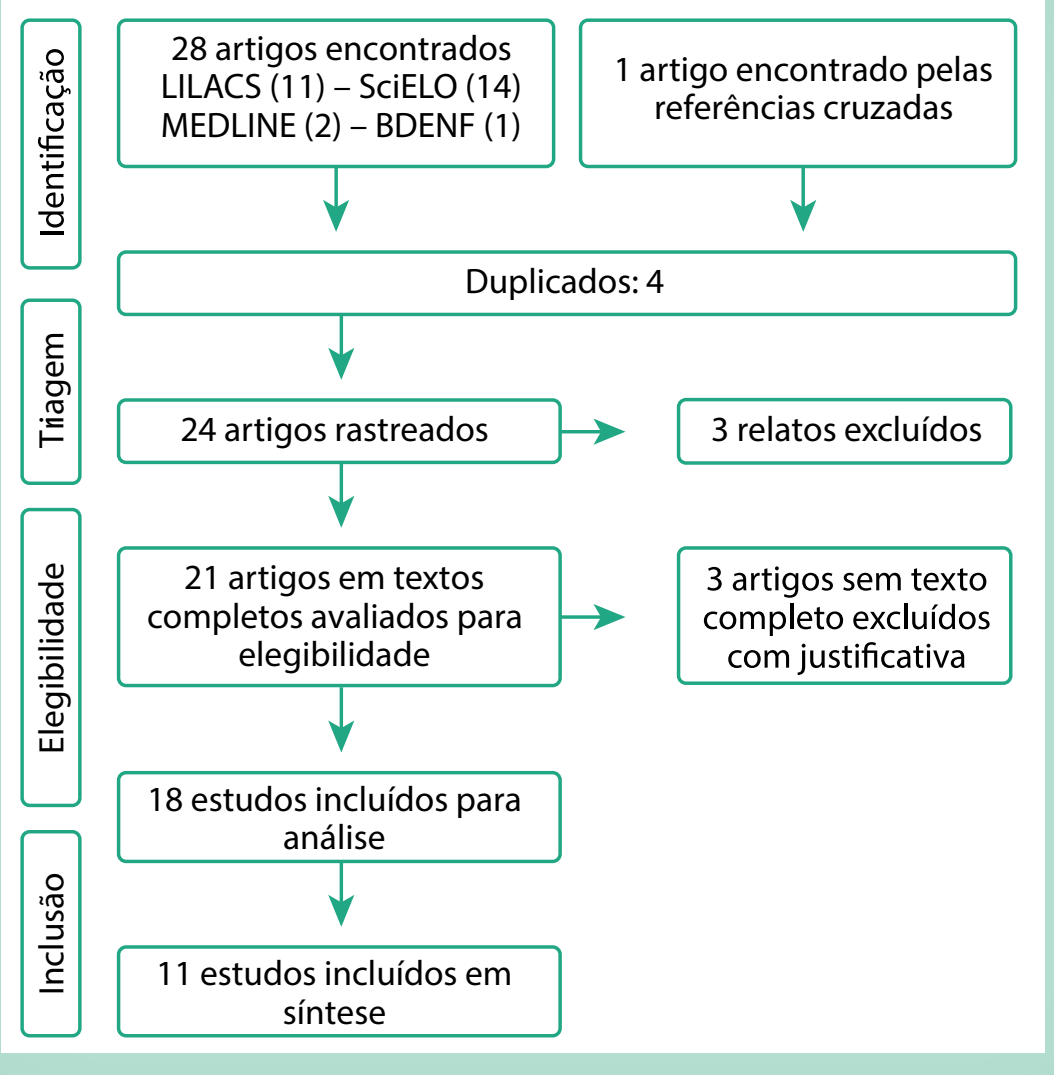

Fonte: Elaborada pelas autoras (2020).

Tabela 1 - Fatores que dificultam o aleitamento materno exclusivo, 2014-2019.

\section{Fatores}

n \%

Uso de mamadeiras, complementares e chupetas

$5 \quad 85$

Grau de escolaridade

$4 \quad 68$

Fator socioeconômico

$4 \quad 68$

Estado emocional das mães

351

Tipo de parto

351

Mães que trabalham fora

351

Falta de preparo dos profissionais

351

Culturas e crenças já vivenciadas pelas mães na amamentação

234

Experiências estressantes no parto

234

Depressão pós-parto

234

Insegurança de amamentar após a alta da maternidade e falta da rede de apoio

234

Início do pré-natal tardio

234

Não realização do aleitamento materno na primeira hora de vida

234

Falta de apoio e incentivo por parte do pai ou parceiro

$2 \quad 34$

Informações incoerentes e contrárias das recomendadas pelo guia alimentar do MS (jovens); início do pré-natal tardio; bebês pré-termo; leite materno fraco; uso de mamadeira e complementares; insegurança para amamentar; fator socioeconômico; depressão pós-parto; falta de conhecimento das políticas públicas pelas mães; falta do alojamento conjunto; experiências estressantes no parto; informações erradas contidas na internet; fragmentação dos serviços de saúde; não valorização dos conhecimentos das mães pelos profissionais; e práticas e rotinas das instituições (Tabela 1).

A Figura1 apresenta os 11 artigos analisados, considerando o ano e o autor, os objetivos, a população do estudo, seguida de sua média de idade, e os fatores de risco. A descrição detalhada das oito intervenções educativas analisadas, considerando os desfechos esperado e alcançado, foi organizada de maneira descritiva.

Segundo um estudo de caso e controle(16), as amostras da pesquisa foram de conveniência e,com base nestas, foram aplicados testes estatísticos, que correlacionavam os níveis socioeconômicos com o AMI.Os resultados mostram que, quanto menor o nível socioeconômico, menor é a adesão do AMI.

Um estudo ${ }^{(17)}$ de corte transversal realizado em 2010, durante uma campanha de vacinação, foi composto de 2.259 binômios mães-crianças,aos quais foram aplicados a Escala de Depressão Pós-Parto de Edimburgo, instrumento utilizado para o rastreamento da depressão puerperal, e um formulário com perguntas sobre características sócio demográficas da família, atenção ao pré-natal, parto e puerpério, sintomas de depressão pós-parto e saúde da criança. O resultado mostrou maior prevalência da ausência da AME em crianças cujas mães apresentavam depressão pós-parto e início do pré-natal tardio. Estas mães eram adolescentes e com fator socioeconômico de baixa renda.

Em um estudo ${ }^{(19)}$ do tipo transversal realizado em 2011, durante uma campanha de vacinação, foi aplicada uma amostra por conglomerado, resultando em 1.027 pares de mãe-filho para o estudo. Foi utilizado um formulário, a partir do qual foram 
Valorização de cada mulher no seu papel de nutriz

\begin{tabular}{lcc}
\hline Idade das mães (jovens) & 1 & 17 \\
\hline Falta do alojamento conjunto & 1 & 17 \\
\hline Práticas e rotinas das instituições & 1 & 17 \\
\hline Dificuldade de sucção (dos recém-nascidos que não tiveram a AMPH) & 1 & 17 \\
\hline Limitação à amamentação noturna & 1 & 17 \\
\hline Lesões nos seios & 1 & 17 \\
\hline Qualidade do pré-natal insatisfatória & 1 & 17 \\
\hline Leite materno fraco & 1 & 17 \\
\hline Gestação não planejada & 1 & 17 \\
\hline Ausência de companheiro & 1 & 17 \\
AMPH: aleitamento materno na primeira hora de vida; Ms: Ministério da Saúde. &
\end{tabular}

investigados vários fatores relacionados ao pré-natal, ao parto e à saúde da mulher e da criança. Foram utilizadas, também, as características sócio demográficas das mães e a assistência ao pré-natal, ao parto e ao puerpério. $\mathrm{O}$ resultado identificou que a maioria não fez o pré-natal de forma adequada e que o tipo de parto interfere na realização do $\mathrm{AMPH}$, sendo que as mães e os filhos que não permaneceram em alojamento conjunto após o parto tiveram a redução da probabilidade da AMPH. Fatores como experiência estressante no parto, falta de preparo dos profissionais, práticas e rotinas institucionais e estado emocional das mães prejudicaram a realização do aleitamento materno.

Em um estudo descritivo e comparativo(20), executado em 2014, foram analisados 50 sites classificados como não científicos e que divulgaram informações sobre alimentação para crianças menores de dois anos. O resultado do estudo mostrou uma discordância das informações dos sites, visitados por pais e cuidadores, em relação ao que é preconizado pelo MS. As mais discrepantes foram sobre a alimentação complementar.

Um estudo analítico ${ }^{(21)}$ desenvolvido com 71 puérperas ainda na maternidade, tendo a coleta de dados realizada por dois questionários (um socioeconômico e outro sobre conhecimento referente ao aleitamento materno), apresentou como resultado que puérperas com maior renda familiar tenderam a ter um maior percen- tual de conhecimento sobre o tema e que o estado emocional das mães interfere diretamente no desmame precoce. Apesar de entender sobre o aleitamento materno,a maior parte das puérperas utiliza, de acordo com o estudo, mamadeiras e complementos por crença e hábito de outra gestação. Além disso, algumas ainda acreditam que o leite materno é fraco.

Um estudo ${ }^{(22)}$ de pesquisa descritiva e prospectiva e com abordagem quantitativa, realizado no $2^{\circ}$ e $3^{\circ}$ trimestres de 2015, em uma instituição com a IHAC, de Foz do Iguaçu (PR), município de Tríplice Fronteira, junto com Porto Iguaçu (Argentina) e Cidade do Leste (Paraguai),mostrou subsídios para que profissionais de saúde e gestores hospitalares refletissem sobre as práticas do AMPH e investissem no aprimoramento de ações para potencializar os benefícios na atenção à saúde materna e infantil.A realização da AMPH mostrou melhor adaptação da sucção quando comparada àqueles que não amamentaram na primeira hora, trazendo, com isso, o uso de complementos e mostrando, também, que o parto vaginal foi um fator protetor para o AMPH.

$\mathrm{O}$ artigo ${ }^{(25)}$ sobre um estudo transversal com aplicação de questionário para mães e avaliação antropométrica de 324 crianças buscou desenvolver um escore nas inadequações alimentar e sua relação com variáveis socioeconômicas, clínico- epidemiológicas e nutricionais. Os resultados indicaram que grande parte das mães apresen- tou baixa escolaridade(38,3\%) e baixa renda familiar(71,1\%), vivem sem companheiro $(22,5 \%)$, são as provedoras da casa (19,5\%), não possuem casa própria (36,7\%), recebem apoio do governo(28,1\%) e fizeram oito ou mais consultas de pré-natal $(69,9 \%)$. No entanto, mais metade das mães realizaram o parto cesariano. Isso provavelmente justifica os altos índices de bebês prematuros e de baixo peso ao nascerem. Fator de risco: fatores socioeconômicos.

$\mathrm{O}$ artigo ${ }^{(26)}$ apresenta um estudo longitudinal baseado em coorte de nascimentos, desenvolvido com 168 puérperas.Foram realizadas três entrevistas - aos 30, 60 e 120 dias após o parto - com o objetivo de avaliar os determinantes ao abandono do AME. A depressão pós-parto e o parto traumático tiveram relação direta com o abandono do aleitamento materno no segundo mês após o parto. Outras variáveis vieram mais adiante, como menor escolaridade, não possuir imóvel próprio, ter de voltar a trabalhar e falta de orientações no puerpério. A variável "não recebeu ajuda do companheiro com a criança" apareceu depois do quarto mês após o parto. Fator de risco: depressão pós-parto.

Em um estudo randomizado ${ }^{(23)}$, profissionais de 20 Unidades de Saúde receberam orientações quanto às diretrizes alimentares para lactentes do MS.E, aos seis meses de idade da criança, realizou-se uma visita domiciliar para obtenção de variáveis relacionadas ao aleitamento materno e à introdução de novos alimentos. Foram 619 crianças: 318 no grupo de intervenção e 301 no grupo-controle. Houve prevalência de aleitamento materno no primeiro $(72,3 \% \times 59,4 \%)$, no segundo $(62,6 \%$ x48,2\%) e no terceiro mês de vida ( $44 \%$ x $34,6 \%$ ). A prevalência foi maior no grupo de intervenção do que no grupo-controle. A prevalência de crianças que consumiram carne quatro ou mais vezes na semana foi superior no grupo de intervenção. A prevalência de crianças que já haviam consumido refrigerante, chocolate, petitsuisseecafé nos primeiros seis meses de vida foi menor no grupo de intervenção. Fator de risco: Falta de preparo dos profissionais. 
Em um estudo ${ }^{(24)}$ de coorte com envolvimento de 1.344 pares (mães e filhos) selecionados em maternidades em Feira de Santana (BA), estes foram acompanhados durante seis meses por meio de visitas domiciliares e com registro da interrupção do AME.Para tanto, a duração mediana foi estimada usando a curva de sobrevida de Kaplan-Meier. O objetivo deste estudo foi identificar fatores associados à cessação precoce da AME em uma cidade localizada no Nordeste brasileiro. A duração mediana da AME foi de 89 dias, e as variáveis que compuseram este resultado foram: a apreciação da mãe parceira pela amamentação e a limitação do número de mamadas noturnas. No entanto, as variáveis que nunca foram descritas, mas que se mostraram associadas foram presença de rachaduras nos mamilos e pré-natal prestado pelo serviço público. Outras variáveis que mostraram associação com o desfecho foram orientações sobre o aleitamento materno recebido no hospital e nascimento em um hospital amigo da criança. Fator de risco: Falta de preparo dos profissionais.

Em um estudo(18) qualitativo fenomenológico, baseado na teoria dos valores de Max Scheler, realizado entre maio e junho de 2014, em alojamentos conjuntos de dois hospitais universitários do Rio de Janeiro e do Rio Grande do Sul, e do qual participaram 20 nutrizes, os dados foram organizados e submetidos à técnica de análise compreensiva e interpretados segundo a teoria dos valores e as políticas específicas de aleitamento materno. O objetivo foi analisar a valoração axiológica da mulher/nutriz quanto ao manejo clínico da amamentação. E, como resultados, emergiram duas unidades temáticas: o manejo clínico da amamentação e seu valor vital e a rede de saúde como apoio no cuidado à amamentação: um valor utilitário. Fator de risco: falta de preparo dos profissionais.

\section{DISCUSSÃO}

O processo de amamentação é uma etapa de grandes transformações e desafios para as mães, além de ser um momento de enorme importância para a saúde do bebê. $\mathrm{O}$ aleitamento materno pode ser considerado um formador de vínculo entre mãe e filho, pois é um processo de reconhecimento entre ambos. No entanto, é um período que envolve diversos sentimentos e emoções, e é relevante enxergar cada mãe individualmente nessa fase.

A prática da amamentação pode ser influenciada por diversos fatores, conforme apresentados nos resultados. Os estudos mostraram que a saúde mental das mães é um fator de grande relevância, já que diversos fatores podem ser desencadeados por meio do estado emocional delas, como a depressão pós-parto, que é definida pela OMS como uma condição de profunda tristeza, desespero e falta de esperança que acontece logo após o parto. A depressão pós-parto traz inúmeras consequências ao vínculo da mãe com o bebê, sobretudo no que se refere ao aspecto afetivo. Este fator acaba comprometendo a garantia do AME e colaboram para o uso de mamadeiras, chupetas e complementares antes dos seis meses de vida, pois o estado emocional das mães é um fator determinante, tanto na produção quanto na escassez de leite. E, quando a saúde mental dessas mulheres está comprometida, isso acaba afetando a sua produção, fazendo com que introduzam complementos precocemente.

Faz-se necessária uma rede de apoio que possa oferecer as mães uma assistência com uma abordagem psicológica, humanizada, acolhedora, sem julgamentos e que irá preservar a autonomia delas, tanto no pré-parto quanto no pós-parto, para lidar com as inseguranças esperadas neste período e, assim, preservar o desenvolvimento do bebê. ${ }^{(27-28)}$

Quanto aos fatores de risco - tais com otipo de parto, experiência estressante no parto, falta de alojamento conjunto e práticas e rotinas das instituições-, estes estão entrelaçados, pois, conforme apresentado na literatura ${ }^{(19)}$, o tipo de parto tem grande interferência na realização da amamentação, principalmente na $\mathrm{AMPH}$, já que a cesárea influencia no seu adiamento, por fatores como a anestesia e o próprio procedimento cirúrgico. Uma dificul- dade também encontrada na $\mathrm{AMPH}$, segundo a literatura ${ }^{(24)}$, são as lesões nos seios, que contribuem para que não ocorra a amamentação nesse primeiro instante.

O estudo constatou que as experiências estressantes no parto podem interferir no início do aleitamento materno, e, muitas vezes, as práticas e rotinas das instituições fazem com que a protagonista principal tenha pouca ou nenhuma autonomia no poder de decisão. Portanto, há a necessidade de se avaliar com maior critério a adesão à cesárea, uma vez que, de acordo com a OMS, as taxas de cesarianas têm aumentado repetidamente, sem nenhum benefício para as mulheres. A nova recomendação feita pela OMS engloba opiniões, medos e crenças de mulheres e profissionais da área da Saúde sobre a cesárea. Cabe aos profissionais trabalharem, com a sua equipe, intervenções educacionais no atendimento às gestantes, parturientes e puérperas e fazerem com que essas mulheres se tornem instruídas sobre os seus direitos e sejam protagonistas das suas decisões. É importante também a conscientização dos profissionais e gestores em promover e estimular a AMPH, já que essa etapa é fundamental para o êxito do aleitamento materno.

Na maior parte dos artigos, o fator socioeconômico mostrou-se como um dos grandes dificultadores. Segundo a literatura ${ }^{(29)}$, a análise da influência dessa condição pode apresentar um caráter dicotômico,que se dá por um elemento dividido em duas partes, geralmente opostas e que se contrapõem.

Além disso, o estudo ${ }^{(30)}$ constatou que famílias de baixo nível socioeconômico não teriam um grande risco de interromper a AME, o que pode ser justificado pela falta de condições econômicas em obter substituto do leite materno. Por outro lado, essas pessoas que se encontram em um nível socioeconômico baixo podem interromper a $\mathrm{AME}$ por falta de conhecimento e instruções.

$\mathrm{Na}$ mesma forma, o estudo ${ }^{(17)}$ relata que famílias de alto nível socioeconômico apontam o risco maior de substituição do leite materno por outros complementos, já que elas têm maior acesso a esses substitutos. Em contrapartida, apresentam maior 
grau de instrução, o que seria um facilitador para a percepção dos benefícios da amamentação. Desta forma, faz-se indispensável à conscientização da população, independentemente da sua classe socioeconômica, cabendo aos profissionais intervirem de modo que todos se conscientizem e retirem suas dúvidas em relação ao AME.

Para as pesquisadoras do estudo ${ }^{(31)}$ a gravidez não planejada também apresenta grandes riscos para a incorporação da amamentação, uma vez que esse fator pode dificultar o vínculo entre mãe e fiIho. A ausência de companheiro ou a falta de apoio e incentivo da amamentação por parte do pai ou parceiro tem uma enorme relevância para a adesão ao aleitamento materno. O desenvolvimento de ações voltadas à saúde sexual também deve ser trabalhado, e informações sobre métodos contraceptivos devem ter grande destaque na Atenção Primária. Além disso, é importante que os profissionais de Saúde incentivem o planejamento familiar para evitar outras gestações não desejáveis.

A participação do companheiro ou familiar se faz necessária também quando a mãe precisa trabalhar fora. $\mathrm{O}$ estudo ${ }^{(32)}$ comprova que o trabalho gera uma grande mudança na amamentação. Devido à necessidade de voltar a trabalhar, a mãe não consegue continuar com a amamentação da mesma forma e,em muitos casos, tem a diminuição nas durações das mamadas ou até mesmo a interrupção. Além disso, o estresse e a jornada de trabalho ajudam a caminhar rumo ao desmame precoce.

Outra vertente que se encontra como um dificultador é a idade materna, que se destaca por ser um fator que envolve a princípio questões estéticas. No entanto, nota-se que,conforme mencionado anteriormente em um estudo(17) que cita o grau de instrução como um facilitador para a percepção dos benefícios da AME,quanto mais jovens as mães,maiores são os índices de abandono, justificando-se pela insegurança das adolescentes no que se refere à capacidade de amamentar. Também se notou, neste estudo, que mães mais velhas tendem a não interromper a amamentação.
Por falta de experiência e conhecimento, muitas mães acreditam que seu leite materno é fraco e não sustenta a criança e, por esta razão, abandonam o AME. Nesta vertente, observa-se que o tema é recorrente e precisa ser mais esclarecido. Um estudo(21) identificou que $15,2 \%$ de mães acreditam que existe leite fraco. Visto isso, observa-se que os profissionais de Enfermagem têm a importante função de instruir essas mães e levar maior segurança em relação às informações, já que, segundo o guia alimentar do MS, a descida do leite (apojadura) ocorre entre o $3^{\circ}$ e o $5^{\circ}$ dias e, por razões de crença ou valores, as mães interpretam erroneamente essas informações.

O pré-natal bem feito é um elemento essencial para o sucesso dos cuidados com o bebê em relação ao AME. No estudo ${ }^{(19)}$, a maioria da amostra não fez o pré-natal de maneira adequada $(78,4 \%)$, porém, na literatura estudada para fundamentar a pesquisa, a satisfação da mulher e o sucesso da amamentação estão relacionados ao número de consultas realizadas, bem como com o acoIhimento da equipe. Há indicativos de que este cenário possa melhorar, porém deve-se levar em conta a integralidade da assistência prestada e o reconhecimento de seus direitos à saúde, bem como a necessidade de promoção da cidadania e participação social das usuárias. No pré-natal, é criado um vínculo, construído durante as consultas. $\mathrm{E}$ isso é um importante quesito para o atendimento humanizado da atenção. Dessa maneira,é possível conseguir a adesão, a satisfação e a permanência da gestante no acompanhamento do pré-natal na saúde pública.

Os profissionais de Enfermagem articulam entre os vários níveis de assistência à gestante. Assim, alguns autores ${ }^{(23-24)}$ referenciam em seus estudos a importância do preparo destes profissionais para que a puérpera se sinta segura e valorizada em seu papel de nutriz. As diferentes razões que as levam a buscar os serviços de saúde, tanto no período pré quanto no pós-natal, estão relacionadas à escuta adequada dos profissionais, que incorporam ações educativas como base da saúde do binômio mãe-filho que devem ser desenvolvidas por todos os profissionais que integram a equipe da Unidade de Saúde. A equipe multidisciplinar deve se comprometer a resolver problemas advindos desse período, e essa relação deve ser pautada em parâmetros humanitários e de solidariedade.

Deste modo, o enfermeiro deve buscar sempre se qualificar e se embasar em conhecimentos para que possa prestar uma assistência de qualidade, enaltecendo os princípios do Sistema Único de Saúde (SUS) e trabalhando com toda a equipe para sempre ter um olhar mais humanizado. Ele deve levar para a população o conhecimento sobre os direitos e deveres e trazê-la para mais perto do serviço de Saúde.

Como limitação deste estudo, destaca-se o pouco número de artigos localizados que abordam a temática. O foco do estudo foram publicações nacionais, com o objetivo de se aproximar mais da realidade brasileira.

\section{CONCLUSÃO}

A partir deste estudo, podem ser observados fatores que favorecem a dificuldade do processo do AME, o que acaba gerando a sua interrupção. Os fatores evidenciados e que mostraram maior destaque no estudo foram o uso de mamadeiras e complementares, o grau de escolaridade das mães e o fator socioeconômico. Para enfrentar esses e os outros fatores que foram encontrados no estudo, é importante a participação e orientação dos profissionais de Enfermagem, visto que o apoio e a segurança que os profissionais fornecem para essas mães, principalmente no pré-natal, têm grande influência na execução da prática do aleitamento materno.

O pré-natal, destacado no estudo, é o momento mais oportuno para esclarecer dúvidas e prestar às gestantes mais informações sobre o tema. Portanto, é indispensável a execução de um excelente pré-natal, que não se limite ao básico e consiga envolver não somente a mãe neste processo, mas também o parceiro e a família.

Faz-se necessário também o apoio profissional no âmbito hospitalar após o nascimento, tanto para a mãe quanto para o 
recém-nascido. Com uma assistência de qualidade, a mãe poderá executar seu papel como protagonista principal e sair da maternidade sem receios e incertezas.

$\mathrm{O}$ aleitamento materno, por mais natu-

ral que aparenta ser, necessita de cuidados e orientações. Assim, ressalta-se a importância dos profissionais de Enfermagem para a promoção do AME.

\section{Referências}

1. Rodrigues WFG, Candeia RMS, Tavares JS, Santos MCS, Andrade KG, Rodrigues BFL. Basic care notebooks: child health, maternal breast and supplementary feeding. Rev Enferm UFPE on line. 2018;12(1):280-2. DOI: https://doi.org/10.5205/1981-8963-v1 2i01a230078p280-282-2018.

2. Hay G, Bærug AB. Fordel med fullammingtilseksmåneder. Tidsskrift Nor Legeforen. 2019;1(32):50-4. DOI: http://dx.doi.org/10.4045/tidsskr.19.0105.

3. World Health Organization. Implementation guidance: protecting, promoting and supporting breastfeeding in facilities providing maternity and newborn services: the revised Baby-Friendly Hospital Initiative. Geneva:WHO; 2018. Disponível em: https:/l apps.who.int/iris/handle/10665/272943.

4. Ministério da Saúde (BR). Secretaria de Atenção à Saúde. Departamento de Atenção Básica. Saúde da criança: aleitamento materno e alimentação complementar. 2. ed. Brasilia (DF): MS; 2015. Disponível em: https://bvsms.saude.gov.br/bvs/publicacoes/saude_crianca_aleitamento_materno_cab23.pdf.

5. Schincaglia RM, Oliveira AC, Sousa LM, Martins KA. Práticas alimentares e fatores associados à introdução precoce da alimentação complementar entre crianças menores de seis meses na região noroeste de Goiânia. EpidemiolServ Saúde. 2015;24(3):465-74. DOI: 10.5123/S1679-49742015000300012.

6. Santos AA dos, Resende MA, Maia GP, Carvalho NC de J, Júnior A de PF. 0 papel do enfermeiro na prevenção do desmame precoce. REAEnf [Internet]. 7 fev.2020 [citado 24ago.2020];2:e2232. Disponível em: https://acervomais.com.br/index.php/enfermagem/article/view/2232.

7. Gomes, JMF., et al. Amamentação no Brasil: discurso científico, programas e políticas no século XX. In: PRADO, SD., et al. orgs. Estudos socioculturais em alimentação e saúde: saberes em rede. [online]. Rio de Janeiro: Eduerj, 2016. Sabor metrópole series, v. 5, p. 475-491. ISBN: 978-85-7511-456-8. Disponível em: http://books.scielo. org/id/37nz2/epub/prado-9788575114568.epub. DOl: 10.7476/9788575114568.

8. Ministério da Saúde (BR). Portaria 1.920, de 5 de setembro de 2013. Institui a Estratégia Nacional para Promoção do Aleitamento Materno e Alimentação Complementar Saudável no Sistema Único de Saúde (SUS) - Estratégia Amamenta e Alimenta Brasil. Brasilia: Ministério da Saúde; 2013. Disponível em: http://bvsms.saude. gov.br/bvs/saudelegis/gm/2013/prt1920_05_09_2013.html.

9. Ministério da Saúde (BR). Área Técnica de Saúde da Criança e Aleitamento Materno. Departamento de Ações Programáticas Estratégicas. Secretaria de Atenção à Saúde. Brasilia: Ministério da Saúde; 2010. Disponível em:http://portal.saude.gov.br/ portal/arquivos/pdf/relatorioihacatualizado.pdf.

10. Azevedo ARR, Alves VH, Souza RMP, Rodrigues DP, Branco MBLR, Cruz AFN. Clinical management of breastfeeding: knowledge of nurses. Esc Anna Nery. 2015;19(3):439-45. DOl: http://dx.doi.org/10.5935/1414-8145.20150058.

11. Marinho MDS, Andrade EM, Abrão ACFV. A atuação do(a) enfermeiro(a) na promoção, incentivo e apoio ao aleitamento materno: revisão bibliográfica. RevEnferm Contemp. 2016;4(2):189-98. DOI: 2317-3378rec.v4i2.598.

12. Oliveira CS, locca FA, Carrijo MLR, Garcia RATM. Breastfeeding and complications that contribute to early weaning. Rev Gaúcha Enferm. 2015;36(spe):16-23. DOI: http://dx.doi.org/10.1590/1983-1447.2015.esp.56766.

13. Sardinha D, Maciel D, Gouveia S, Pamplona F, Sardinha L, Carvalho M, Silva A. Promoção do aleitamentomaterno no pré-natal peloenfermeiro. Revista de EnfermagemUFPE on line [Internet]. 16mar2019; [citado 24ago2020]; 13(3):852-857. Disponívelem: https://periodicos.ufpe.br/revistas/revistaenfermagem/article/view/238361. 14. Moher D, Liberati A, Tetzlaff J, Altman DG, Altman DG, The PRISMA Group. Preferred reporting items for systematic reviews and meta-analyses: the PRISMA statement. PLos Med. 2009;6(7):e1000097. DOl:https://doi.org/10.1371/journal. pmed.1000097.

15. Roever L. Compreendendoosestudos de revisãosistemática. Rev Soc Bras Clin Med. 2017 abr-jun;15(2):127-130. Disponívelem: https://fiadmin.bvsalud.org/document/view/r34d2.

16. Patiño-Suárez JT, Campos-Uscanga Y. Las semanas de gestación como fator asociado a lapráctica de lalactancia materna inmediata. AvEnferm. 2018;36(2):153-60.
DOI: http://dx.doi.org/10.15446/av.enferm.v36n2.66822.

17. Silva CS, Lima MC, Sequeira-de-Andrade LAS, Oliveira JS, Monteiro JS, Lima NMS, et al. Association between postpartum depression and the practice of exclusive breastfeeding in the first three months of life. J Pediatr (Rio J). 2017;93(4):356-64. DOI: http://dx.doi.org/10.1016/j.jped.2016.08.005.

18. Alves VH, Padoin SMM, Rodrigues DP, Silva LA, Branco MBLR, Marchiori GRS. Clinical management of breastfeeding: axiological value from women's perspective. Esc Anna Nery. 2016;20(4):e20160100. DOl: https://doi.org/10.5935/14148145.20160100.

19. Bandeira de Sá NN, Gubert MB, Santos W, Santos LMP. Factors related to health services determine breastfeeding within one hour of birth in the Federal District of Brazil, 2011. Rev Bras Epidemiol. 2016;19(3):509-24. DOl: https://doi. org/10.1590/1980-5497201600030004.

20. Monteiro GSG, Assis MM, Leite MA, Mendes LL. Avaliação das informações nutricionais referentes às crianças de até dois anos disponíveis em sites populares. Rev Paul Pediatr. 2016;34(3):287-92. DOl: http://dx.doi.org/10.1016/j.rppede.2016.03.004.

21. BoffADG, Paniagua LM, Scherer S, Goulart BNG. Mother's social/economic aspects and level of knowledge about breastfeeding. AudiolCommun Res. 2015;20(2):141-5. DOl: https://doi.org/10.1590/S2317-64312015000200001517.

22. Netto A, Spohr FA, ZillyA, França AFO, Rocha-Brischiliari SC, Silva RMM. Breastfeeding in the first hour of life at an institution with the baby-friendly hospital initiative. CiencCuidSaúde. 2016;15(3):515-21. DOl: https://doi.org/10.4025/cienccuidsaude.v15i3.31508. 23. Vitolo MR, Louzada MLC, Rauber F. Positive impact of child feeding training program for primary care health professionals: a cluster randomized field trial. Rev Bras Epidemiol. 2014;17(4):873-86. DOl: https://doi.org/10.1590/18094503201400040007.

24. Vieira GO, Reis MR, Vieira TO, Oliveira NF, Silva LR, Giugliani ERJ. Trends in breastfeeding indicators in a city of northeastern Brazil. J Pediatr. 2015;91(3):270-7. DOI: https://doi.org/10.1016/j.jped.2014.08.012.

25. Mais LA, Domene SMÁ, Barbosa MB, Taddei JAAC. Diagnóstico das práticas de alimentação complementar para o matriciamento das ações na Atenção Básica. Ciênc Saúde Coletiva. 2014;19(1,):93-104. DOI: http://dx.doi.org/10.1590/141381232014191.2168.

26. Machado MCM, Assis KF, Oliveira FCC, Ribeiro AQ, Araújo RMA, Cury AF, et al. Determinants of the exclusive breastfeeding abandonment: psychosocial factors. Rev SaúdePública. 2014;48(6):985-94. DOl: https://doi.org/10.1590/s00348910.2014048005340.

27. Manente MV, Rodrigues OMPR. Maternidade e trabalho: associação entre depressão pós-parto, apoio social e satisfação conjugal. Pensando Fam. 2016;20(1):99111. Disponivel em: http://pepsic.bvsalud.org/scielo.php?script=sci_arttext\&pi$d=$ S1679-494X2016000100008\&lng=pt\&tlng=pt.

28. Malekzadeh JM, Synaii S, Koor BE, Falsafian G, Nakhaie MR. Growth indices of exclusively breastfed until 6 months age and formula-fed infants in southwest of Iran. Int J PrevMed. 2019;10(207):1-13. DOI: 10.4103/ijpvm.IJPVM_36_18.

29. Carrascoza KC, Possobon RF, Ambrosano GMB, Costa Jr. ÁL, Moraes ABA. Determinantes do abandono do aleitamento materno exclusivo em crianças assistidas por programa interdisciplinar de promoção à amamentação. Ciênc Saúde Coletiva. 2011;16(10):4139-46. DOI: http://dx.doi.org/10.1590/S1413-81232011001100019. 30. Mascarenhas MLW, Albernaz EP, Silva MB, Silveira RB. Prevalence of exclusive breastfeeding and its determiners in the first 3 months of life in the South of Brazil. J Pediatr. 2006;82(4):289-94.DOI:10.2223/JPED.1506.

31. Conceição SP, Fernandes RAQ. Influence of unintended pregnancy on breastfeeding duration. EscAnna Nery.2015;19(4):600-5. DOI: 10.5935/1414-8145.20150080. 32. Silva AM, Santos MCS, Silva SEM, Ferreira FA, Freitas RSC, Santo, REA, et al. Aleitamento materno exclusivo: empecilhos apresentados por primíparas. Revenferm UFPE online. [internet] dez., 2018; 12(12):3205-11. Disponivel em: https://pesquisa. bvsalud.org/portal/resource/pt/biblio-999669. 